\title{
Quantification of Prostate Deformation due to Needle Insertion during TRUS-guided Biopsy
}

\author{
Tharindu De Silva ${ }^{1,2}$, Aaron Fenster ${ }^{1,2,3}$, \\ Jagath Samarabandu ${ }^{2}$, and Aaron D. Ward ${ }^{1}$ \\ ${ }^{1}$ Imaging Research Laboratories, Robarts Research Institute \\ 2 Biomedical Engineering Graduate Program, University of Western Ontario \\ ${ }^{3}$ Department of Medical Biophysics, University of Western Ontario, \\ London, Ontario, Canada \\ tdesilva@imaging.robarts.ca, ward@robarts.ca
}

\begin{abstract}
Prostate biopsy is the clinical standard for the diagnosis of prostate cancer, and technologies for 3D guidance to targets and recording of biopsy locations are promising approaches to reducing the need for repeated biopsies. In this study, we use image-based non-rigid registration to quantify prostate deformation during needle insertion and biopsy gun firing, in order to provide information useful to the overall assessment of a TRUS-guided biopsy system's expected targeting error. We recorded mean tissue displacements of up to $0.4 \mathrm{~mm}$, accounting for $16 \%$ of the clinically-motivated maximum desired RMS error of a guidance system.
\end{abstract}

\section{Introduction}

Prostate cancer is one of the most common cancers among men, second only to skin cancer [1. Prostate biopsy is currently the standard clinical practice to obtain a definitive diagnosis of cancer. Two-dimensional (2D) transrectal ultrasound (TRUS) is the most common imaging modality used for image guidance in prostate biopsy due to its low cost and high frame rate. Recently, 3D magnetic resonance imaging (MRI) and TRUS-guided biopsy systems [234] have shown promise for the accurate guidance of biopsy needles to predefined targets. Such systems also provide a 3D record of biopsy locations, which is useful in planning targets for subsequent sessions if pathological analysis of biopsy samples reveals the need to rebiopsy the same or nearby targets in a later session. Biopsy target locations can also be determined based on other modalities such as MRI, requiring MRI-US registration. The need for accurate 3D guidance and recording of biopsies is clear; reports have shown that cancer detection rates during second and third biopsy sessions are only $20 \%$ and $7 \%$, respectively, for 2D TRUS-guided biopsy [5].

Since the smallest tumours considered to be clinically significant have volumes of $0.5 \mathrm{~cm}^{3}$ or greater [6, corresponding to a spherical tumour with a radius of approximately $5 \mathrm{~mm}$, the RMS error of a TRUS-guided biopsy system in delivering a needle to a target must be no more than $2.5 \mathrm{~mm}$ in order

T. Jiang et al. (Eds.): MICCAI 2010, Part III, LNCS 6363, pp. $213-220,2010$.
(C) Springer-Verlag Berlin Heidelberg 2010 
to biopsy clinically significant tumours $95 \%$ of the time. There can be multiple potential sources of error that can cause the actual target biopsy location to be different from the expected target, including: (1) tolerances in the design and construction of mechanical needle guidance systems, (2) errors in imaging and calibration to the needle guidance systems, (3) patient and prostate motion and deformation during the procedure due to interaction with the TRUS probe and discomfort during biopsy, (4) prostate deformation due to slow biopsy needle insertion in preparation for biopsy gun firing, and (5) prostate deformation due to rapid biopsy needle insertion after firing the biopsy gun. There is previous research in measurement of and correction for the first three sources of error 47. However, to the best of our knowledge, prostate deformation due to needle insertion through the rectal wall and biopsy gun firing has not yet been quantified in the context of prostate biopsy. Although the cumulative effect of all five of the above sources of error has been measured using MRI in the context of a specific MR-compatible robotic biopsy system [8, the specific contributions of needle insertion and biopsy gun firing to the overall error were not reported. In addition, we hypothesize that deformations in response to needle insertion and biopsy gun firing are different in the context of TRUS-guided biopsy due to several important differences in physical configuration. In contrast to the robotic procedure measured in [8], where an endorectal coil in a cylindrical housing is placed parallel to the rectal wall for imaging, TRUS-guided biopsy is typically conducted using an end-firing ultrasound transducer, where the transducer tip is manipulated against the anterior rectal wall in order to obtain images. The MR-guided robot in [8] inserts needles into the prostate through the rectal wall at an oblique angle to the endorectal coil housing, whereas in end-firing TRUS biopsy, the needles are parallel to the probe axis. It is reasonable to expect that these differences in physical configuration may lead to differing mechanical dynamics at the time of biopsy needle insertion and gun firing, resulting in different prostate deformation characteristics. The effect of needle insertion on prostate motion has been studied extensively in the context of brachytherapy procedures 910, where the patient is under genereal anesthesia and the brachytherapy needles are inserted slowly (relative to the firing speed of a biopsy gun) through the perineum. We hypothesize that the effect of the needle in the context of biopsy is different due to the reactions (e.g. in the form of pelvic floor muscle contractions) of an awake, uncomfortable patient and the high speed of needle insertion by the biopsy gun.

In this work, we apply intensity-based, non-rigid image registration to measure the deformation observed in the prostate during (1) the manual insertion of the biopsy needle into the prostate, and (2) the firing of the biopsy gun to acquire the tissue sample during hand-held, 2D TRUS-guided biopsy procedures. At a highlevel, our procedure is as follows: (1) acquire video-rate sequences of 2D TRUS images during the acquisition of each prostate biopsy sample from a series of clinical biopsy sessions; (2) for each biopsy, select three frames, occurring before needle insertion, before biopsy gun firing, and after biopsy gun firing; (3) nonrigidly register these three frames together, validating the registration method 
by measurement of its target registration error (TRE); and (4) quantify prostate deformation by taking clinically relevant measurements of the deformation vector fields given by the registration algorithm.

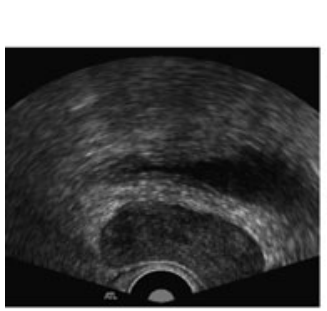

Before needle insertion

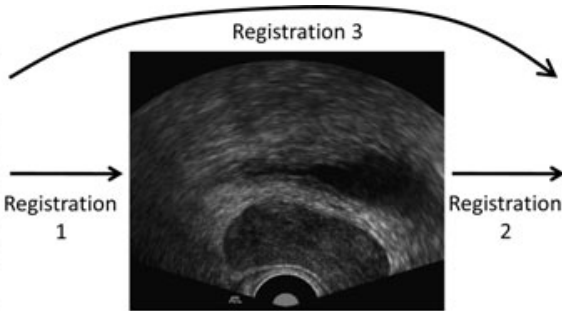

Before biopsy gun firing

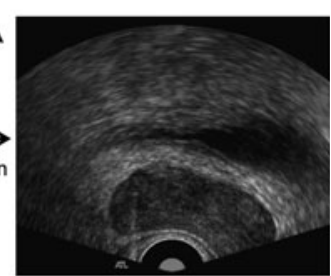

After biopsy gun firing

Fig. 1. Images in a biopsy sequence, and the names used for the three indicated registrations throughout this paper. In all of our images, the needle is to the left of the transducer, as shown in the rightmost image.

\section{Method}

Using a hand-held 2-dimensional (2D) transrectal ultrasound (TRUS) probe, we acquired TRUS images at 30 frames per second during clinical TRUS-guided prostate biopsy sessions of 9 patients ( 3 by one physician and 6 by another) . From each biopsy sample taken, we extracted 3 frames; we refer to each of these frame triplets as a "biopsy sequence". A biopsy sequence (figure 1) consists of: (1) the frame occurring immediately prior to the physician's insertion of the needle through the rectal wall, (2) the frame occurring immediately prior to the firing of the biopsy gun, and (3) the frame occurring immediately after the firing of the biopsy gun. From our 9 patients, we obtained 96 biopsy sequences; an example of a sequence is given in figure 1 .

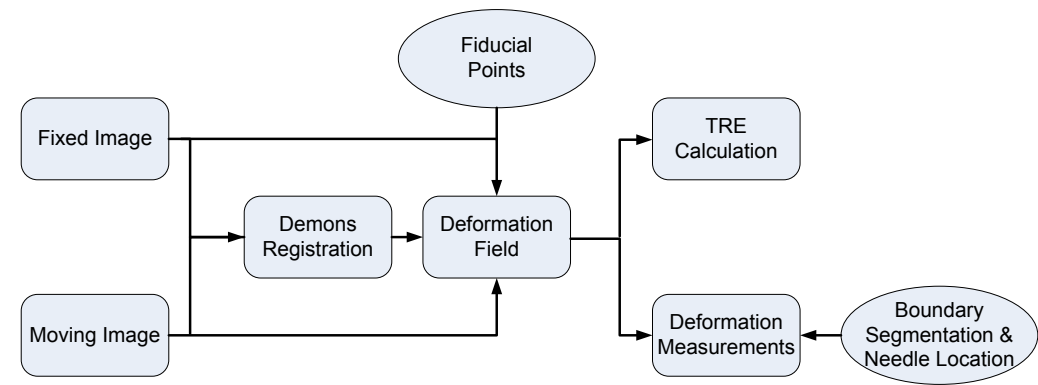

Fig. 2. High level method used in this paper 


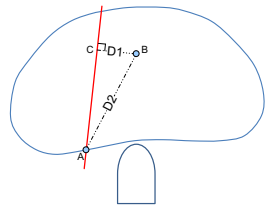

(a)

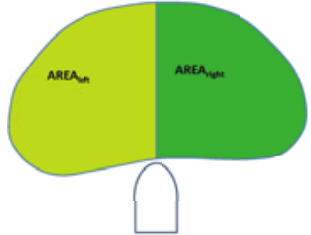

(b)

Fig. 3. (a) Distance from the needle axis (D1) and distance to the piercing point (D2). (b) Area to the left and right of the prostate from the probe axis.

Our method is given at a high level in the block diagram shown in figure 2 . From each biopsy sequence, we choose a pair of images, with the image from the earlier time point designated as the moving image, and the image from the later time point designated as the fixed image. We then nonrigidly register the moving image to the fixed image, yielding a deformation field describing the motion and deformation of the prostate from the earlier time point to the later time point. The deformation field consists of vectors, each having two components that describe the magnitude and the direction of the displacement at each location. In order to validate the chosen registration algorithm, we measure the target registration error (TRE) of manually marked, intrinsic fiducials (calcifications), using the method described in [7]. The spatial relationship between the needle and probe is fixed, and we oriented all the images in this study such that the needle lies at the same location in image space, to the left of the probe. We quantified the deformation fields given by registration using the following clinically-relevant calculations: (1) the mean deformation as a function of distance to the biopsy needle (distance D1 in figure 3(a)), (2) the mean deformation as a function of distance to the piercing point where the needle enters the prostate (distance D2 in figure $3(\mathrm{a})$ ), and (3) the mean deformation as a function of the lateral position of the probe relative to the prostate, determined using the ratio of prostate areas to the left and right of the transducer (figure $3(\mathrm{~b})$ ). The latter two measurements require a prostate segmentation, which we perform manually in this study.

Our immediate use of non-rigid registration in figure 2 (i.e. without performing an initial rigid alignment) is due to our observation that rigid motion of the prostate within the biopsy sequences is minimal (mean rigid misalignment of $0.2 \mathrm{~mm}$ translation and 1 degree rotation). It is also intuitive that needle insertion and gun firing cause local deformations within the prostate. Therefore, we directly use a non-rigid registration algorithm having sufficient flexibility to measure these local deformations. A sample deformation vector field generated by the registration of an image prior to needle insertion to an image immediately after gun firing is shown in figure 4. Figure 4(a) represents the magnitudes of the deformation field vectors, including a manually contoured boundary of the prostate and location of the needle. Figure 4(b) and (c) represent the lateral and axial deformation fields respectively, to illustrate the anisotropy of the deformation field with respect to the orientation of the biopsy needle. 


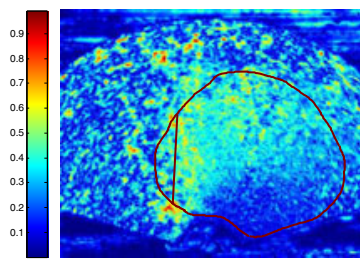

(a)

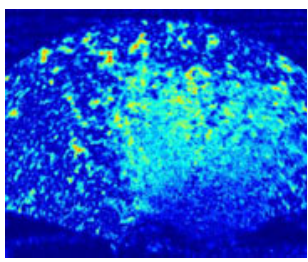

(b)

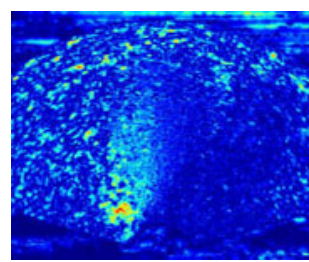

(c)

Fig. 4. (a) Deformation field vector magnitude with prostate boundary and needle location indicated. (b) Lateral (x) components of the deformation field (c) Axial (y) components of the deformation field.

\section{Results}

The TREs of our tested non-rigid registration algorithms are given in table 1. All tested algorithms improved the TRE, with the Demons algorithm providing the best overall results. However, it is worthy of note that the fiducial localization error (FLE) for trans-rectal ultrasound (TRUS) images has been determined in previous work to be $0.2 \mathrm{~mm}$ [7, which indicates that our reported TREs are approaching their lower bound. Nevertheless, the Demons algorithm was chosen for use in this study because (1) it provided the best overall TRE, (2) it is based on optical flow. Optical flow techniques have been shown to be useful in tracking fine-scale structure in ultrasound images in the presence of small tissue motion [11, which is expected in our study where tissue motion is localized in the vicinity of the needle.

Table 1. Comparison of TRE before and after registration

\begin{tabular}{lll}
\hline Registration Method & Registration 1 TRE $(\mathrm{mm})$ & Registration 2 TRE $(\mathrm{mm})$ \\
\hline Before registration & 0.31 & 0.21 \\
Demons & 0.29 & 0.14 \\
Symmetric forces demons & 0.32 & 0.16 \\
Bspline & 0.28 & 0.16 \\
\hline
\end{tabular}

Figure 5(a) shows the relationship between the mean deformation magnitude and the perpendicular distance from the line of the needle insertion within the prostate for Registration 3. The curve plateaus to the right at a mean deformation value of approximately $0.28 \mathrm{~mm}$. Figure 6] decomposes the deformation down into needle insertion (Registration 1) and gun firing (Registration 2) separately. Figure 5(b) shows the mean deformation magnitude as a function of the distance of the deformation vector from the insertion point of the needle into the prostate in Registration 3. Since we are measuring very small deformations, a valid concern is the potential effect of any noise present in the images on our measurements of the deformation fields. Figures 5 and 6 show the means of the unsigned vector magnitudes, i.e., $\frac{1}{N} \sum_{i=1}^{N} \sqrt{x_{i}^{2}+y_{i}^{2}}$, which accumulate the 
contributions of noise throughout the vector field, and may account for the unexpected positive plateau at approximately $0.28 \mathrm{~mm}$. To address this issue, we show in figure 7(a) (corresponding to figure 5(a)) the magnitudes of the signed means of the vector components, i.e., $\sqrt{\left(\frac{1}{N} \sum_{i=1}^{N} x_{i}\right)^{2}+\left(\frac{1}{N} \sum_{i=1}^{N} y_{i}\right)^{2}}$. Similarly, figure 7(b) gives the corresponding information to figure 5(b). We take the signed means of the vector components under the assumption that the true tissue motion is regular and any noise is irregular, with a tendency to self-cancellation when taking the signed mean. This effect is illustrated by the lower plateaus observed in figure 7. To explore the relationship between deformation and the relative positions of the probe and the prostate, we plotted the mean deformation magnitude of vectors within $5 \mathrm{~mm}$ of the needle (which is the clinical region of interest for biopsy targeting) versus the ratio of areas within the prostate to the left of the probe axis and to the right of the probe axis in figure 8 , The point on the $\mathrm{x}$ axis at 0.5 depicts the situation where the probe is aligned with the central axis of the prostate. We used linear regression to fit a line to the resulting cluster of points; the correlation ratio was found to be -0.15 .

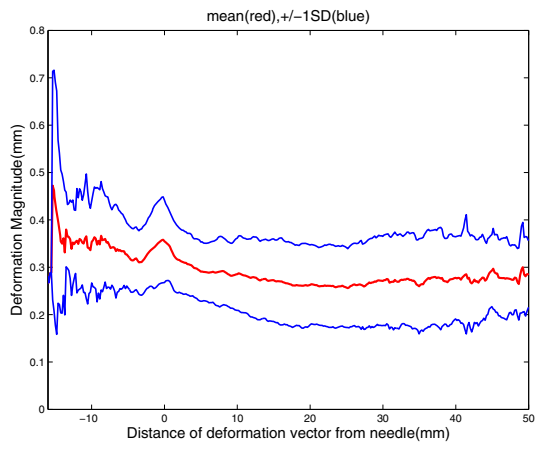

(a)

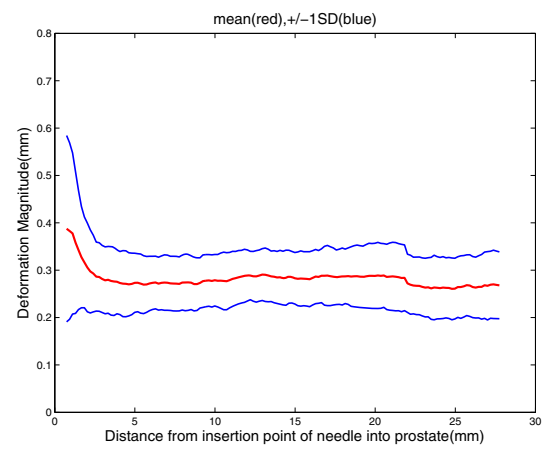

(b)

Fig. 5. Mean (red) and +/- 1 standard deviation (blue) of unsigned deformation vector field magnitudes for Registration 3, (a) as a function of distance from the needle, and (b) as a function of distance to the piercing point within the prostate

\section{Discussion}

In the graph in figure 5(a), the deformation to the left of the probe (nearer to the prostate edge) is approximately $0.1 \mathrm{~mm}$ higher than to the right. There is a slight trend of increased magnitude of deformation toward both edges of the prostate. Figure 6 decomposes this deformation into needle insertion (Registration 1) and gun firing (Registration 2) separately. The shapes of the two graphs in figure 6 are similar to that obtained for Registration 3 (figure 5(a)). However, note that the deformation after gun firing is approximately $0.1 \mathrm{~mm}$ higher than that due to needle insertion before gun firing. This shows that a significant portion of the deformation of Registration 3 (figure 5(a)) is due the firing of the gun. 


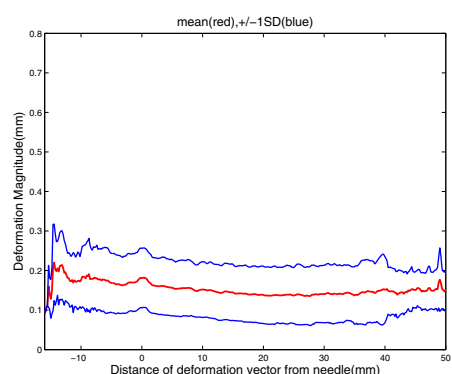

(a)

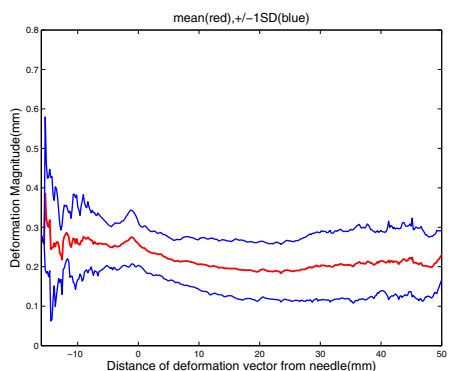

(b)

Fig. 6. Mean (red) and $+/-1$ standard deviation (blue) of unsigned deformation vector field magnitudes as a function of distance from the needle for (a) Registration 1, (b) Registration 2

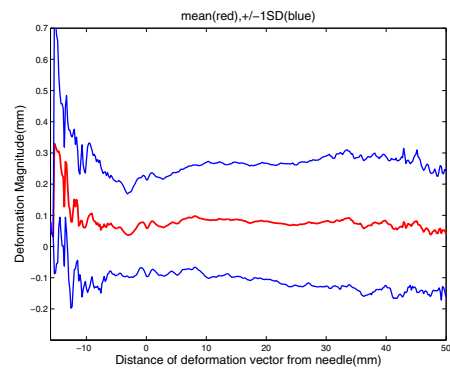

(a)

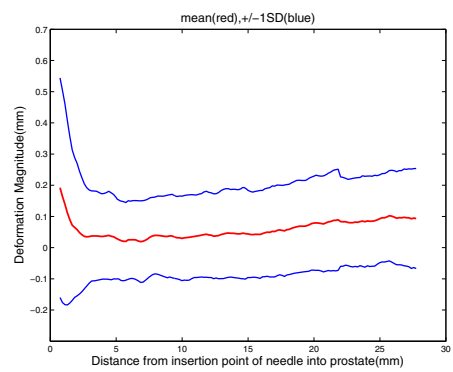

(b)

Fig. 7. Mean (red) and +/- 1 standard deviation (blue) of the magnitudes of the signed vector component means for Registration 3, (a) as a function of distance from the needle, and (b) as a function of distance to the piercing point within the prostate

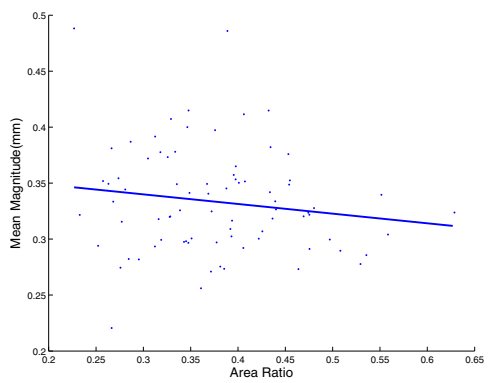

Fig. 8. Deformation magnitude as a function of the left:right (relative to the transducer centerline) ratio of prostate areas

Figure 5(b) shows a concentration of deformation within $5 \mathrm{~mm}$ of the insertion point of the needle into the prostate. At the piercing point, the deformation is approximately $0.4 \mathrm{~mm}$. The deformation at the piercing point is about 0.2 $\mathrm{mm}$ higher than the deformation in the surrounding region. Registration 1 and 
Registration 2 also showed a similar trend and the deformation was larger in Registration 2 (biopsy gun firing) than in Registration 1.

\section{Conclusion}

Observed mean tissue displacements as a consequence of needle insertion and gun firing are as high as $0.4 \mathrm{~mm}$. These displacements could account for $10 \%$ $20 \%$ of the allowable $2.5 \mathrm{~mm}$ RMS error permitted in a biopsy system designed to successfully sample spherical targets with $5 \mathrm{~mm}$ radius $95 \%$ of the time. We observe that the majority of the deformation due to needle insertion and biopsy gun firing occurs lateral to the needle, away from the center of the prostate, and at the needle's piercing point at the prostate boundary.

\section{References}

1. Canadian Cancer Society's steering committee: Canadian cancer statistics 2009 (2009)

2. Cool, D., Sherebrin, S., Izawa, J., Chin, J., Fenster, A.: Design and evaluation of a 3D transrectal ultrasound prostate biopsy system. Medical Physics 35(10), 4695-4707 (2008)

3. Krieger, A., Susil, R.C., Ménard, C., Coleman, J.A., Fichtinger, G., Atalar, E., Whitcomb, L.L.: Design of a novel MRI compatible manipulator for image guided prostate interventions. IEEE Transactions on Biomedical Engineering 52(2), 306313 (2005)

4. Bax, J., Cool, D., Gardi, L., Knight, K., Smith, D., Montreuil, J., Sherebrin, S., Romagnoli, C., Fenster, A.: Mechanically assisted 3D ultrasound guided prostate biopsy system. Medical Physics 35(12), 5397-5410 (2008)

5. Keetch, D.W., Catalona, W.J., Smith, D.S.: Serial prostatic biopsies in men with persistently elevated serum prostate specific antigen values. The Journal of Urology 151(6), 1571-1574 (1994); PMID: 7514690

6. Epstein, J.I., Sanderson, H., Carter, H.B., Scharfstein, D.O.: Utility of saturation biopsy to predict insignificant cancer at radical prostatectomy. Urology 66(2), 356360 (2005); PMID: 16040085

7. Karnik, V.V., Fenster, A., Bax, J., Cool, D.W., Gardi, L., Gyacskov, I., Romagnoli, C., Ward, A.D.: Assessment of image registration accuracy in three-dimensional transrectal ultrasound guided prostate biopsy. Medical Physics 37(2), 802-813 (2010)

8. Xu, H., Lasso, A., Vikal, S., Guion, P., Krieger, A., Kaushal, A., Whitcomb, L.L., Fichtinger, G.: Accuracy validation for MRI-guided robotic prostate biopsy. In: Medical Imaging, San Diego, California, USA, vol. 7625, pp. 762517-8. SPIE, San Jose (2010)

9. Lagerburg, V., Moerland, M.A., Lagendijk, J.J., Battermann, J.J.: Measurement of prostate rotation during insertion of needles for brachytherapy. Radiotherapy and Oncology 77(3), 318-323 (2005)

10. Stone, N.N., Roy, J., Hong, S., Lo, Y., Stock, R.G.: Prostate gland motion and deformation caused by needle placement during brachytherapy. Brachytherapy 1(3), 154-160 (2002)

11. Meunier, J.: Tissue motion assessment from 3D echographic speckle tracking. Physics in Medicine and Biology 43, 1241-1254 (1998) 